IBS

\section{Self-help interventions in irritable bowel syndrome}

\section{A P S Hungin}

\section{Self-management approach in irritable bowel syndrome was effective in reducing primary care consultations and perceived symptom severity}

W

hat if someone offered you a simple, accessible, and low cost management for irritable bowel syndrome (IBS) which offered results? And moreover, all that was needed was an initial clinical diagnosis without going through the strictures of research led definitions? This is what is proposed by Robinson and colleagues ${ }^{1}$ in this issue of Gut-an outwardly simple study backed by complex prior work with IBS sufferers (see page 643).

In a randomised study, IBS sufferers were enrolled in one of three interventions: a guidebook on IBS, the booklet plus a self-help group session, or management as usual. Set in primary care, the study was pragmatic, relying on the clinicians' own diagnosis and not stipulating any specific diagnostic criteria. The results indicated that the guidebook group did as well as the guidebook plus self-help group and both were better than care as usual. A substantial reduction in primary care consultations was achieved. Although there were no changes in symptom scores in any of the groups, both groups using the guidebook reported a reduction in perceived symptom severity.

This study poses a number of intriguing questions. Of course the guidebook was no mere handy booklet prepared by a keen clinician-it was the result of prior qualitative research with IBS patients who described the information they required to help them cope with their symptoms better. ${ }^{2}$ It can thus be seen as rooted in patients' own perceptions and beliefs and dealing with their likely concerns. None the less, both the apparent lack of adjunctive effect from the group therapy (although it might well be that the effect in those who attended was more pronounced) and the fact that only a minority (40\%) attended their session are worth considering. And what does it mean when patients say they are better but their specific symptoms are not?

Explaining peoples' response to nonpharmacological interventions probably has to do with their personal concepts of health and their health seeking behaviour. ${ }^{3}$ Some people, highly aware and informed about things that can go wrong (the so- called "high monitors") are prone to seek advice early and often. Equally, those who have a lower general concern about health and lower perceptions of worrying pathology (the "low monitors") are less likely to consult. Within these groups, people sometimes comprise the "low blunters" (those whose perceptions and anxiety cause them to have a low threshold for consultation) and their opposites, the "high blunters" (classically, doctors themselves who, while aware of the potential consequences of their own symptoms, will avoid consultation as far as possible). (Blunting is defined as the tendency to under react to symptoms.)

A possible reason why patients might be able to modify or in pragmatic terms reduce their consultation behaviour for conditions that do not have dangerous consequences is through increased knowledge and understanding of their symptoms. This is likely to go some way towards allowing them to live with their symptoms. Much psychological intervention is geared to this and it would seem that a well researched tool, such as a guidebook, can serve that function.

Non-pharmacological interventions in IBS have not become as common practice as some might have hoped. In a critique of psychological treatments, Talley and colleagues ${ }^{5}$ indicated that although the majority of studies reported psychological treatments to be superior to control therapy, the overall efficacy of such treatments could not be established because of methodological inadequacies. On the other hand, Svedlund, ${ }^{6}$ in a review of 22 studies that used a controlled design comparing psychological treatment with conventional medical treatment and/or supportive therapy, concluded that psychological treatment appeared superior and that psychotherapy was an efficient complement to drugs. Recently, in a trial of cognitive behavioural therapy delivered by primary care nurses, Kennedy et al showed additional benefit over mebeverine alone for up to six months. ${ }^{7}$ The methodological problems referred to often centre on the control or "placebo" comparison used. Even in drug intervention trials for IBS the role and indeed the definition of placebo continues to cause confusion. Against a backdrop of placebo effects ranging from $16 \%$ to $70 \%$, ${ }^{8}$ identifying the exact role of the pharmaceutical intervention is challenging.

Where then do the psychological interventions come in against this backdrop? Some extremists might go so far as to say that the psychological intervention itself might be a placebo and that such interventions will naturally produce a measurable effect. However, harnessing this effect represents an important therapeutic tool. As Enck and Klosterhalfen ${ }^{9}$ point out, high placebo responses in functional bowel disorders are similar to those in non gastrointestinal diseases and even "not too dissimilar" to those in organic gastrointestinal disorders, such as duodenal ulcer and inflammatory bowel disease. Furthermore, psychobiological mechanisms of the placebo response can be identified in brain function studies such as imaging. Clinicians frequently use therapeutic approaches which seem to work even if they do not understand how they work and even if they know that their approach is probably no different from what might be regarded as placebo.

However, not everyone will want to avail themselves of certain types of psychological intervention. A low level of participation in groups is known to happen when sensitive or embarrassing topics are being explored. Researchers collecting information are familiar with the dilemma of having to decide whether alternative techniques, such as individual interviews, might be more successful under these circumstances. Those participating in self-help groups for such conditions (and IBS is a sensitive issue for many sufferers $)^{10}$ are likely to be selfselected having overcome their apprehensions or are more desperate.

The guidebook concept, as described by Robinson and colleagues, ${ }^{1}$ is an attractive one. An instrument derived from patients' own perceptions and experiences elevates it beyond an information tool alone and probably constitutes a psychological intervention in its own right. This team has also demonstrated that a self-management approach in inflammatory bowel disease was effective in reducing hospital visits and increased sufferers' confidence. ${ }^{11}$ Similar interventions, perhaps utilising other media such as video or interactive information technology, may offer more promise than might be anticipated if 
they are based on well researched constructs and offer meaningful information. Tools that help the busy everyday generalist, particularly the primary care physician, and which do not have significant time and resource implications, can provide firstline management for many IBS sufferers. Equally, studies that are not hamstrung by unnecessarily reductionist approaches, rendering them remotely applicable to the clinical settings, should be welcomed.

Gut 2006;55:603-604.

doi: 10.1136/gut.2005.075606

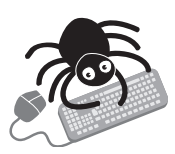

Conflict of interest declared (the declaration can be viewed on the Gut website at http://www.gutjnl.com/ supplement).
Correspondence to: Professor A P S Hungin, Centre for Integrated Health Care Research, Wolfson Research Institute, University of Durham, Queen's Campus, Stockton on Tees TS17 6BH, UK; A.P.S.Hungin@durham.ac.uk

\section{REFERENCES}

1 Robinson A, Lee V, Kennedy A, et al. A randomised controlled trial of self-help interventions in patients with a primary care diagnosis of irritable bowel syndrome. Gut 2006:55:643-8.

2 Kennedy AP, Robinson A, Rogers A. Incorporating patients' views and experiences of life with IBS in the development of an evidence based self-help guidebook. Patient Educ Couns 2003;50:303-10.

3 Miller SM, Brody DS, Summerton J. Styles of coping with threat: implications for health. J Pers Soc Psychol 1988;54:142-8.

4 Ingham JG, Miller P. Self referral to primary care: symptoms and social factors. J Psychosom Res 1986;30:49-56.

5 Talley NJ, Owen BK, Boyce P, et al. Psychological treatments for irritable bowel syndrome: a critique of controlled treatment trials. Am J Gastroenterol 1996;91:277-83

6 Svedlund J. Functional gastrointestinal diseases. Psychotherapy is an efficient component to drug therapy. Lakartidningen 2002;17:172-4.

7 Kennedy T, Jones R, Darnley S, et al. Cognitive behaviour therapy in addition to antispasmodic treatment for irritable bowel syndrome: randomised controlled trial. BMJ 2005;331:435.

8 Patel SM, Statson WB, Legedza A, et al. The placebo effect in irritable bowel syndrome trials: a meta-analysis. Neurogastroenterol Motil 2005; 17:332-40.

9 Enck P, Klosterhalfen S. The placebo response in functional bowel disorders: perspectives and putative mechanisms. Neurogastroenterol Motil 2005; 17:325-31.

10 Hungin APS, Whorwell PJ, Tack J, et al. The prevalence, patterns and impact of IBS: an international survey of 40,000 subjects. Aliment Pharmacol Ther 2003; 17:643-50.

11 Kennedy P, Nelson E, Reves D, et al. A randomised controlled trial to assess the effectiveness and cost of a patient orientated self management approach to chronic inflammatory bowel disease. Gut 2004;53:1639-45.

Colitis

\section{T regulatory cell suppression of colitis: the role of TGF- $\beta$}

\section{R Duchmann, M Zeitz}

Transforming growth factor $\beta$ (TGF- $\beta$ ) and interleukin 2 may be involved in IBD peripheral regulatory T cell pathophysiology, raising the possibility of therapeutic application of TGF- $\beta$ induced regulatory T cells in IBD patients

C $\mathrm{D} 4+\mathrm{CD} 25+$ regulatory $\mathrm{T}$ cells $\left(\mathrm{T}_{\text {reg }}\right)$ expressing the lineage marker Foxp3 control immune responses to self- and foreign antigens and are an intensely studied member of the heterogenous group of regulatory $\mathrm{T}$ cells. Although initially described as a population of suppressor $\mathrm{T}$ cells required to avoid organ specific autoimmunity, it has subsequently become clear that $\mathrm{T}_{\text {reg }}$ control immune responses in a much broader sense, including transplantation tolerance and immune responses to pathogens and tumours. ${ }^{1-3}$ Relevant to inflammatory bowel diseases (IBD), $\mathrm{T}_{\text {reg }}$ prevent $^{4}$ and treat established ${ }^{5}$ colitis in animal models of IBD and are numerically deficient in patients with active IBD. ${ }^{6}$ This underlines the fact that the immune dys-equilibrium characteristic of chronic inflammatory diseases involves concomitant disturbances in inflammatory and suppressive immune mechanisms and opens up novel approaches for IBD therapy by strengthening $\mathrm{T}_{\mathrm{reg}}$ mediated suppression.
Currently, efforts in laboratories worldwide are addressing the central questions of $\mathrm{T}_{\text {reg }}$ immunology, resolution of which will help us see more clearly the role of $\mathrm{T}_{\text {reg }}$ in disease pathogenesis and therapy. Some of these central questions are: where do $\mathrm{T}_{\text {reg }}$ come from; how are they generated, expanded, and maintained; how and where do they function; why do they fail to control immune responses in disease; and how can their therapeutic potential be used most efficiently and safely. As current data indicate that $\mathrm{T}_{\text {reg }}$ from IBD patients are functionally normal ${ }^{6-8}$ but numerically deficient during active disease, ${ }^{6}$ it will be interesting to understand the underlying mechanisms and, based on that knowledge, devise the most promising strategy to enlarge their numbers to therapeutic levels.

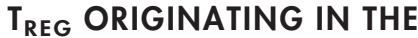 THYMUS}

It was first described that during ontogeny, $\mathrm{CD} 4+\mathrm{CD} 25+\mathrm{T}_{\text {reg }}$ originate in the thymus, become detectable in the periphery of normal mice from around day 4 after birth, and increase to adult numbers by week 3. ${ }^{9}$ Production of Foxp3 expressing $\mathrm{T}_{\mathrm{reg}}$ by the thymus is considerably delayed relative to nonregulatory thymocytes, ${ }^{10}$ and neonatally thymectomised mice show an early and substantial reduction in peripheral CD4+CD25+ $\mathrm{T}_{\text {reg, }}$ associated with the development of autoimmune diseases. It was demonstrated in transforming growth factor $\beta 1$ (TGF $\beta 1)^{11}$ and interleukin 2 (IL-2) ${ }^{12}$ deficient mice, that both cytokines, in contrast with their important function on mature peripheral $\mathrm{T}_{\text {reg, }}$ are dispensable for intrathymic $\mathrm{T}_{\text {reg }}$ development. Efficient generation of CD4+CD25+ thymocytes results from relatively high affinity interaction of the $\mathrm{T}$ cell receptor (TCR) with agonist ligands expressed in thymic epithelial cells. ${ }^{13}{ }^{14}$ This process requires $\mathrm{CD} 28$ dependent costimulation of developing thymocytes $^{15}$ and thymic stromal lymphopoietin expressed by human Hassall's corpuscles to activate thymic CDllct dendritic cells to express high levels of CD80 and CD86. ${ }^{16}$ Whether a thymic defect contributes to $\mathrm{T}_{\text {reg }}$ pathophysiology in IBD is not known. Interestingly, however, thymic $\mathrm{T}_{\text {reg }}$ are functionally impaired in patients with autoimmune myasthenia gravis, ${ }^{17}$ and it was shown in animal models that colitis induces aberrant thymic development with impaired $\mathrm{T}_{\text {reg }}$ cell production. ${ }^{18}$

\section{$T_{\text {REG }}$ ORIGINATING IN THE PERIPHERY}

An important next step in our understanding of $\mathrm{T}_{\text {reg }}$ biology was when it was shown that Foxp3+ T cells with regulatory function in vivo do not only 